\title{
Determinação da umidade do solo por micro-ondas e estufa em três texturas de um Latossolo Vermelho-Amarelo do Cerrado
}

\author{
Luis Fernando Cunha de Oliveira ${ }^{1}$, Cassiano Garcia Roque ${ }^{1}$ \\ ${ }^{1}$ Universidade Federal de Mato Grosso do Sul - UFMS, Câmpus de Chapadão do Sul, Chapadão do Sul, Mato Grosso do Sul, Brasil. \\ E-mail: fcunhaoliver@hotmail.com, cassiano.roque@ufms.br
}

Recebido: 12/06/2016; Aceito: 31/10/2016.

\section{RESUMO}

O objetivo do trabalho foi estudar a equivalência de tempos de secagem para determinação de umidade do solo em forno micro-ondas comparada ao método padrão (estufa). Tal estudo foi realizado em um Latossolo Vermelho-Amarelo de Cerrado nas classes texturais de 20, 35 e 50\% de argila. As amostras de solo foram submetidas a cinco tempos de secagem no micro-ondas $(2 ; 4 ; 6 ; 8$ e 10 minutos) em comparação com o método padrão $\left(110{ }^{\circ} \mathrm{C} / 24 \mathrm{~h}\right)$, em um experimento inteiramente casualizado. As análises de regressão dos dados quantitativos apresentaram altos coeficientes de determinação, demonstrando a possibilidade do uso do microondas na determinação da umidade do solo (\%) pela rapidez e confiabilidade de resultados em relação ao padrão de estufa.

Palavras-chave: curvas de umidade, método alternativo, padrão.

\section{Determination of soil moisture in microwave and standard method in three textures a Red- Yellow Latosol of Cerrado}

\begin{abstract}
The objective of this study was to investigate the equivalence of drying times of soil samples for determination of its moisture in the microwave oven compared to the standard method (drying oven). This study was conducted in a Red-Yellow Latosol of Cerrado in the textural classes of 20,35 and 50\% clay. The study samples were subjected to drying five times in the microwave $(2 ; 4 ; 6 ; 8: 10$ minutes $)$ compared to the standard method $\left(110{ }^{\circ} \mathrm{C} /\right.$ $24 \mathrm{~h}$ ). The results demonstrated the possibility of using microwaves in determining soil moisture (\%) for the speed and reliability of results compared to standard oven.
\end{abstract}

Key words: moisture curve; alternative method; standard. 


\section{Introdução}

Os Latossolos ocupam $46 \%$ das áreas do Cerrado. Nessa área, são representados por Latossolos vermelhoamarelo e vermelho escuro. Em sua maior parte, apresentam baixa capacidade de retenção de água. Tal aspecto aliado a alta radiação solar, alta evapotranspiração potencial e ocorrência de veranicos nos Cerrados, condicionam que a água seja um recurso que exige cuidados especiais à agricultura na região (COCHRANE et al., 1985). Isso pode ser limitante, pois a água afeta diversas propriedades do solo e o desenvolvimento das plantas (REICHARDT; TIMM, 2004).

Dessa forma, variações na umidade do solo influenciam a disponibilidade de nutrientes, a atividade de microrganismos e a adoção de práticas culturais de manejo do solo, entre outros. Por causa disso, a estimativa correta da umidade do solo é de suma importância para as operações mecanizadas e para as avaliações das propriedades do solo, particularmente, na região considerada deste estudo.

O método tradicional usado para a determinação da umidade do solo é o de estufa (padrão). O procedimento consiste na secagem de uma amostra em forno convencional (estufa) numa temperatura de $110^{\circ} \mathrm{C} \pm 5$ ${ }^{\circ} \mathrm{C}$, em um intervalo de 24 horas (EMBRAPA, 1997). Nesse método, as amostras de solo, podem ser retiradas em vários locais e profundidades no campo, na forma simples ou composta, deformada ou indeformada, estas últimas retiradas com trados especiais.

O principal inconveniente da estufa é o longo tempo de secagem do solo. Para resolver isso, diversos métodos têm sido experimentados. Um deles é o uso do forno micro-ondas, onde a secagem do solo pode ser realizada de forma rápida e com menor consumo de energia. Essa justificativa é reforçada por Miller et al. (2007), para quem o uso de micro-ondas propicia redução significativa do tempo de secagem da amostra comparado ao método de estufa. Este tempo será uma função do número de amostras, do tamanho e potência do forno, do teor de umidade inicial, da massa da amostra e do tipo de solo.

Souza et al. (2002) observam que o micro-ondas constitui uma forma alternativa e mais rápida do que o método convencional para secagem do solo e da umidade da planta; entre 10 e 14 minutos obtêm-se o resultado.

O processo de secagem em micro-ondas está baseado no fenômeno de que as moléculas de água quando expostas a radiação eletromagnética, sofrem rotação, liberando calor com maior rapidez (KINGSTON et al., 1997). No entanto, para obter resultados corretos é necessário calcular a potência dos equipamentos.
Na prática, existe diferença entre a potência máxima, normalmente de $700-800 \mathrm{~W}$ indicada pelos fabricantes, e a potência efetiva. Dessa forma, antes de realizar qualquer teste experimental é necessário realizar a calibração do micro-ondas, para determinar o valor da temperatura e da potência real de trabalho do aparelho (BARBOZA et al., 2001).

Adotando esse cuidado, as determinações de umidade do solo de plantas e de grãos armazenados têm sido satisfatórias. Em outras aplicações na agricultura, a irradiação de micro-ondas pode provocar alterações nos resultados de análises químicas e mineralógicas (TOMÉ; DECHEN, 1995).

Considerando a confiabilidade dos resultados obtidos, este trabalho teve como objetivo estabelecer a equivalência de tempos de secagem (em minutos) para determinação de umidade do solo pelo forno de microondas em relação ao método padrão de estufa em diferentes classes texturais de um Latossolo VermelhoAmarelo de Cerrado.

\section{Material e Métodos}

As amostras foram obtidas em Latossolo VermelhoAmarelo distrófico de topografia plana com texturas argilosa $(50 \%)$, franca-argilosa $(35 \%)$ e franca- arenosa (20\% de argila), com coordenadas geográficas de Lat (18.1387) e Long (-53.2354) na parte mais arenosa da área de estudo, e Lat (-18.1365), Long (-53.2438) na textura mais argilosa.

As classes texturais foram caracterizadas através de pontos georreferenciados na área em questão. A amostragem de campo foi realizada com trado mecânico de rosca de 7/8 polegadas, na profundidade de 0 a 0,20 m. Após a coleta, as amostras foram hermeticamente fechadas em sacos plásticos, e armazenadas em refrigerador pelo prazo de 24 horas, para evitar perdas de umidade.

$\mathrm{Na}$ determinação da umidade do solo, as amostras com $100 \mathrm{~g}$ de massa das três classes texturais foram submetidas a cinco tratamentos em micro-ondas: TM2 (2 min); TM4 (4 min); TM6 (6 min); TM8 (8 min) e TM10 (10 min) e comparadas ao padrão de estufa TE $\left(110{ }^{\circ} \mathrm{C} / 24 \mathrm{~h}\right)$. O delineamento experimental foi inteiramente casualizado, com cinco repetições por tratamento, totalizando 25 unidades amostrais. Para determinação da umidade pelo método padrão (estufa), seguiu-se a metodologia usual (EMBRAPA, 1997).

A determinação da umidade do solo em micro-ondas foi realizada em modelo com potência nominal de $800 \mathrm{~W}$, tensão de alimentação $127 \mathrm{v}-60 \mathrm{~Hz}$, freqüência de micro-ondas de $2,450 \mathrm{MHz}$ e consumo de $1,3 \mathrm{Kw} / \mathrm{h}$. As medições foram feitas com potência única (100\%). Os resultados de umidade do solo (\%) foram expressos 
em base seca (UBS), de acordo com a equação da umidade gravimétrica preconizada por Klein (2008).

$$
U_{G}(\%)=\underline{M}_{\underline{u}} \underline{-} \underline{M}_{S} \cdot 100 / M_{S}
$$

onde, $\mathrm{UG}=$ umidade gravimétrica em \% de massa seca; $\mathrm{UM}=$ massa úmida da $\operatorname{amostra}(\mathrm{g}) ; \mathrm{MS}=$ massa seca da amostra (g).

Após o completo resfriamento das amostras, a massa seca foi determinada em balança com precisão de $0,01 \mathrm{~g}$. Para a calibração do micro-ondas foram realizados os seguintes procedimentos indicados por Souza et al. (2002): 1) Foi calibrado um volume de água na temperatura ambiente $\left(23 \pm 2^{\circ} \mathrm{C}\right) ; 2$ ) Foi pesado $1 \mathrm{~kg}$ de água e disposta em uma jarra plástica; 3) Foi medida a temperatura da água $(\mathrm{TI})$ - recomendada em $23 \pm 2{ }^{\circ} \mathrm{C}$; 4) A água foi disposta no prato de aquecimento do micro-ondas durante 2 minutos na potência indicada como máxima (100\%); 5) Após, retirou-se o recipiente, agitou-se a água e registrou-se a temperatura final (TF), no máximo, 30 segundos após o aquecimento; 6) Foi substituída água aquecida por outra na temperatura ambiente; 7) O procedimento foi repetido para 80, 60, 40 e $20 \%$ da potência máxima; 8) Foram realizadas três medidas a cada potência; 9) Por último, calculou-se a potência real de trabalho pela equação (2):

$$
P=35(T F-T I) \text {. }
$$

onde, $\mathrm{P}=$ Potência real de trabalho para cada situação em W.J.seg ${ }^{-1}(100,80,60,40$ e $20 \%$ da potência máxima; $\mathrm{TF}=$ temperatura final em ${ }^{\circ} \mathrm{C} ; \mathrm{TI}=$ temperatura inicial em ${ }^{\circ} \mathrm{C}$.

Aplicando-se essa metodologia ao tempo de secagem inicial de dois minutos em amostra de um litro de água, na potência máxima (P10) indicada de 800W, obteve-se uma potência real de trabalho no valor de $700 \mathrm{~W}$ do equipamento utilizado no estudo. Por tratar-se de dados quantitativos, as amostras de solo foram submetidas à análise de regressão quadrática com um nível de confiança superior a $95 \%(a=0,05)$.

Para determinar os valores de igualdade dos tempos de secagem das amostras de solo em micro-ondas, nas diferentes texturas em relação à estufa, foi realizada análise de regressão, cujas equações de segundo grau foram calculadas pela fórmula universal de Bháskara (3), descrita abaixo.

$\boldsymbol{Y}=\boldsymbol{a} \boldsymbol{x}^{2}+\boldsymbol{b x}+\boldsymbol{c}$, onde a, b e c são coeficientes transpostos da equação de segundo grau para cálculo da expressão abaixo,

$$
x=\frac{-b \pm \sqrt{b^{2}-4 a c}}{2 a}
$$

\section{Resultados e Discussão}

A estatística descritiva das amostras de umidades do solo mostra claramente que a média da umidade final após a secagem das amostras apresenta diferenças entres as classes, conforme pode ser visto abaixo (Tabela 1).

As diferenças obtidas nas médias finais de umidades de solo indicam que tais resultados podem ser atribuídos ao teor inicial de água e ao percentual de argila em cada amostra dentro das respectivas classes texturais, com diferenças na capacidade de retenção de água. Por esta razão, diversos autores afirmam que não se deve comparar a umidade do solo de diferentes locais, texturas e profundidade de amostragem.

Além disso, verifica-se na mesma tabela que os tratamentos de 8 e 10 minutos em micro-ondas para as diferentes texturas retiraram quantidades de água superiores ao padrão estufa, muito embora as diferenças observadas na textura média e arenosa não sejam estatisticamente significativas.

Esse fato talvez indique que o método de microondas tenha retirado água do solo acima do potencial mátrico, isto é, tenha superestimado a umidade de campo, sem que as plantas possam retirar essa porção correspondente de água presente no solo.

Na tabela, a umidade média de solo foi obtida com amostras de $100 \mathrm{~g}$ de massa. Cremon et al. (2014) constataram que amostras de $40 \mathrm{~g}$ apresentaram melhor efeito de secagem do que as de 20 e 60 g. Neste trabalho, verificou-se que o comportamento da umidade gravimétrica apresentou não só diferenças entre as classes de textura, mas também segundo o tempo progressivo de secagem, conforme demonstra as figuras abaixo.

Pela observação dos valores médios de umidade do solo das Figuras (1, 2 e 3 ) o comportamento das variáveis de umidade gravimétrica e tempo de secagem aumentou progressivamente, quase que em uma regressão linear, traduzido por coeficientes de determinação próximos a um $(1,0)$, conforme se observa nas equações de regressão determinadas para cada classe de textura.

Os valores interpolados pela conhecida fórmula de Bháskara nas equações quadráticas para igualar os tempos de secagem das amostras em micro-ondas à estufa, foram de 6 min para as texturas argilosa e média, com $50 \%$ e $35 \%$ de argila, e de 7 minutos para amostras de textura arenosa (20\% de argila)

As diferenças obtidas nas médias finais de umidades de solo indicam que tais resultados podem ser atribuídos ao teor inicial de água e ao percentual de argila em cada amostra dentro das respectivas classes texturais, com diferenças na capacidade de retenção de água. Por esta razão, diversos autores afirmam que não deve comparar a umidade do solo de diferentes locais, texturas e profundidade de amostragem. 
Tabela 1. Estatística descritiva das determinações de umidade de solo conforme a classe textural e tratamentos em micro-ondas

\begin{tabular}{|c|c|c|c|c|c|c|}
\hline Tratamentos & Mínimo & Máximo & Mediana & Média & Desvio Padrão & $\mathrm{CV}(\%)$ \\
\hline \multicolumn{7}{|c|}{ Argila: $50 \%$} \\
\hline T02 & 5,82 & 10,49 & 7,52 & 7,82 & 1,84 & 23,50 \\
\hline T04 & 16,27 & 20,48 & 18,34 & 18,36 & 1,51 & 8,21 \\
\hline T06 & 23,03 & 25,39 & 23,83 & 24,04 & 0,86 & 3,56 \\
\hline T08 & 25,05 & 26,02 & 25,40 & 25,53 & 0,38 & 1,48 \\
\hline $\mathrm{T} 10$ & 26,59 & 26,98 & 26,74 & 26,78 & 0,19 & 0,70 \\
\hline $\mathrm{TE}$ & 22,43 & 22,97 & 22,72 & 22,72 & 0,19 & 0,85 \\
\hline \multicolumn{7}{|c|}{ Argila: $35 \%$} \\
\hline T02 & 4,16 & 6,66 & 5,70 & 5,55 & 0,96 & 17,27 \\
\hline T04 & 11,11 & 11,85 & 11,42 & 11,47 & 0,27 & 2,37 \\
\hline T06 & 12,17 & 12,67 & 12,34 & 12,35 & 0,20 & 1,61 \\
\hline T08 & 12,35 & 12,80 & 12,67 & 12,58 & 0,20 & 1,56 \\
\hline $\mathrm{T} 10$ & 12,73 & 13,31 & 12,99 & 12,99 & 0,23 & 1,80 \\
\hline $\mathrm{TE}$ & 12,13 & 12,24 & 12,19 & 12,19 & 0,04 & 0,33 \\
\hline \multicolumn{7}{|c|}{ Argila: $20 \%$} \\
\hline T02 & 2,56 & 4,05 & 2,98 & 3,14 & 0,63 & 20,23 \\
\hline T04 & 4,71 & 5,26 & 4,82 & 4,97 & 0,27 & 5,46 \\
\hline T06 & 4,98 & 5,54 & 5,25 & 5,25 & 0,22 & 4,23 \\
\hline T08 & 5,26 & 5,82 & 5,61 & 5,61 & 0,23 & 4,03 \\
\hline $\mathrm{T} 10$ & 5,31 & 5,93 & 5,74 & 5,70 & 0,24 & 4,12 \\
\hline $\mathrm{TE}$ & 5,49 & 5,66 & 5,54 & 5,56 & 0,07 & 1,20 \\
\hline
\end{tabular}

T02: secagem em micro-ondas: 2 min; T04: 4 min; T06: 6 min; T08: 8 min: TE: estufa (24h).

Além disso, verifica-se na mesma tabela que os tratamentos de 8 e 10 minutos em micro-ondas para as diferentes texturas retiraram quantidades de água superiores ao padrão estufa, muito embora as diferenças observadas na textura média e arenosa não sejam estatisticamente significativas.

Esse fato talvez indique que o método de microondas tenha retirado água do solo acima do potencial mátrico, isto é, tenha superestimado a umidade de campo, sem que as plantas possam retirar essa porção correspondente de água presente no solo.

Na tabela anterior, a umidade média de solo foi obtida com amostras de $100 \mathrm{~g}$ de massa. Cremon et al., (2014) constataram que amostras de $40 \mathrm{~g}$ apresentaram melhor efeito de secagem do que as de 20 e $60 \mathrm{~g}$. Neste trabalho, verificou-se que o comportamento da umidade gravimétrica apresentou não só diferenças entre as classes de textura, mas também segundo o tempo progressivo de secagem, conforme revelam as figuras abaixo.

Pela observação dos valores médios de umidade do solo das Figuras (1, 2 e 3 ) o comportamento das variáveis de umidade gravimétrica e tempo de secagem aumentou progressivamente, próximo à uma regressão linear, traduzido por coeficientes de determinação próximos a um $(1,0)$, conforme se observa nas equações de regressão determinadas para cada classe de textura.

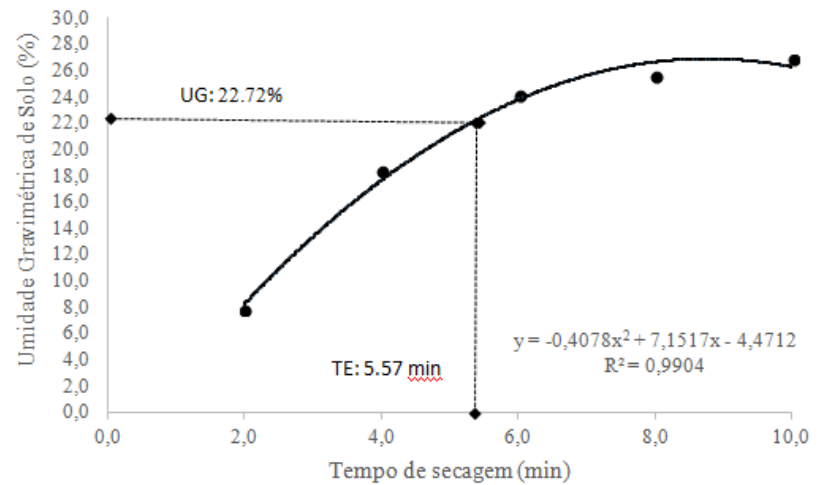

Figura 1. Valores médios de umidade gravimétrica (\%) para amostras com 50\% de argila segundo diferentes tempos de secagem em micro-ondas comparados ao padrão estufa

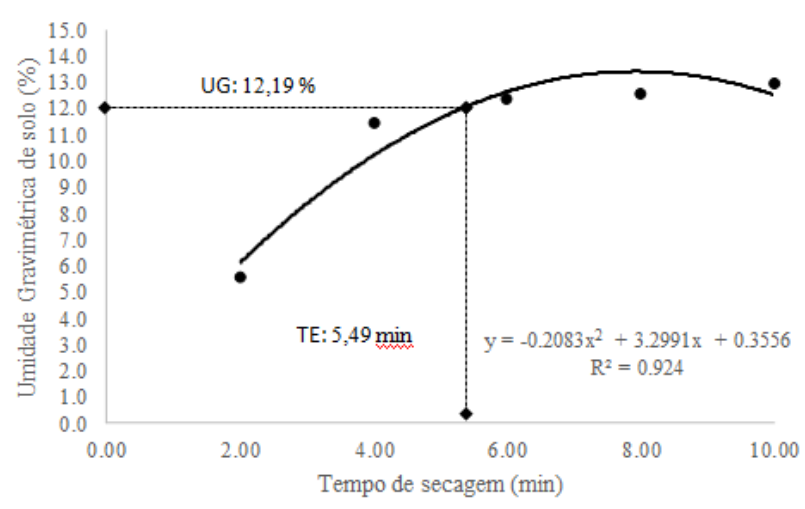

Figura 2. Valores médios de umidade gravimétrica (\%) para amostras com $35 \%$ de argila segundo diferentes tempos de secagem em micro-ondas comparados ao padrão estufa 


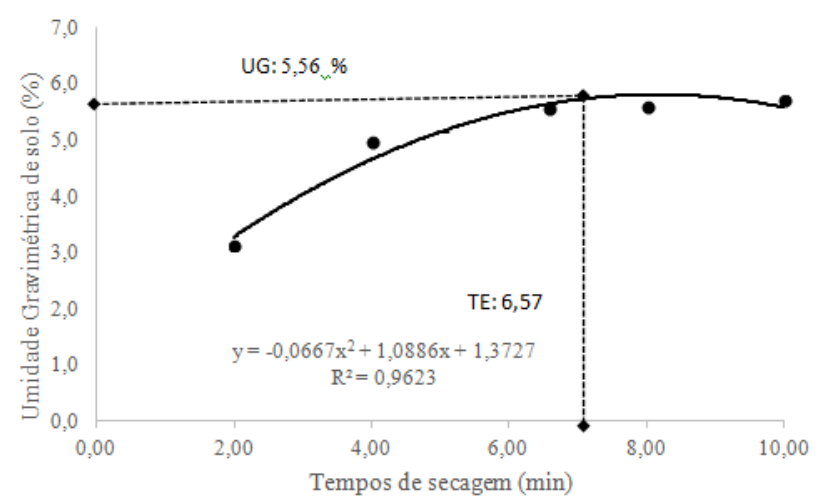

Figura 3. Valores médios de umidade gravimétrica (\%) para amostras com $20 \%$ de argila segundo diferentes tempos de secagem em micro-ondas comparados ao padrão estufa.

Os valores interpolados pela conhecida fórmula de Bhaskara nas equações quadráticas para igualar os tempos de secagem das amostras em micro-ondas à estufa, foram de 6 min para as texturas argilosa e média, com $50 \%$ e $35 \%$ de argila, e de 7 minutos para amostras de textura arenosa (20\% de argila).

A rapidez em que foram obtidos os resultados do forno de micro-ondas em relação ao padrão estufa está baseada propriamente no método de geração de energia, visto que o atrito das moléculas de água resultante do aquecimento rápido no interior do equipamento, gera calor instantâneo, reduzindo a umidade da amostra (AGUILAR, 2001).

Essa diminuição significativa dos tempos de secagem das amostras está em conformidade com trabalhos de Buske et al. (2011), que não observaram diferenças entre tempos de secagem de amostras em micro-ondas de 10 e 15 minutos e tampouco em relação à estufa em análises de três solos do Rio Grande do Sul. Apesar desses resultados similares, Benke e Kearfott (1999) afirmam que o processo de retirada gradativa de água é influenciado pelos teores iniciais de umidade nas amostras de solo.

Dessa forma, não existe uma combinação de tempo e temperatura aceita como padrão, uma vez que o tempo de secagem de amostras para a umidade do solo em micro-ondas é função da quantidade de amostras para secagem, do tamanho da amostra, da umidade inicial, do tipo de solo, de suas texturas e da potência real de trabalho do equipamento.

\section{Conclusões}

O uso de micro-ondas é um método rápido, de fácil uso e eficiente na determinação de umidade de solo. Nas condições do experimento, com amostras de $100 \mathrm{~g}$ e potência real de $700 \mathrm{~W}$ do equipamento utilizado, o total de 7 minutos de equivalência de tempos de secagem das amostras de solo foi suficiente para igualar a umidade do solo em micro-ondas à estufa.

\section{Referências Bibliográficas}

AGUILAR, J. A. G. Procesamiento de materiales por medio de microondas em la FIME. Ingenierías. Nuevo León, v. 4, n. 13, n., p. 32-39, 2001.

BARBOZA, A. C. R. N.; CRUZ, C. V. M. S.; GRAZIANI, M. B.; LORENZETII, M. C. F.; SABADINI, E. Aquecimento em forno de microondas/desenvolvimento de alguns conceitos fundamentais. Quimica Nova, Campinas-SP, v. 24, n. 6, p. 901-904, 2001.

BENKE, R. R.; KEARFOTT, K. J. Soil sample moisture content as a function of time during oven drying for gammaray spectroscopic measurements. Nuclear Instruments and Methods in Physics Research Section A: Accelerators, Spectrometers, Detectors and Associated Equipment, Amsterdam, v. 422, n. 3, p. 817-819, 1999.

BUSKE, T.C; ROBAINA, A. D; PELTER, M. X; BRAGA, F.U.A. Determinação da umidade do solo por diferentes fontes de aquecimento. Irriga, Botucatu-SP, v. 19, n. 02, p. 315 324,2011

CREMON, C.; LONGO, L.; MAPELI, C. N.; SILVA, L. A. M.; SILVA, W. M. Determinação da umidade de diferentes solos do Pantanal Matogrossense via microondas e método padrão. Revista Agrarian, v. 7, n. 24, p. 280-288, 2014.

COCHRANE, T. T.; SANCHEZ, L. G.; AZEVEDO, L. G.; PORRAS, J. A.; GARVER, C. L. Land in tropical América. Cali: CIAT/EMBRAPA-CPAC, 3 v.1985.

EMBRAPA. EMPRESA BRASILEIRA DE PESQUIA AGROPECUÁRIA. Manual de Métodos de análises de solo, $2^{\mathrm{a}}$ ed, Rio de Janeiro-RJ: Centro Nacional de Pesquisa de Solos, 1997, 212p. (Embrapa-CN-Doc:1)

KINGSTON, H. M.; HASWELL S. J. (Ed.) Microwave Enhance de Chemistry Fundamentals, Sample Preparation and Applications. Washington: ACS Professional Reference Book, 1997, 772p

KLEIN, V. A. Física do Solo. Passo Fundo-RS: Universidade de Passo Fundo, 2008. 212p.

MILLER, J. R. J.; SMITH, R. B.; BIGGAR, J. W. Soil water content. Microware method. Proceedings Soil Science Society of America, Madison, v.38, n.3, p. 535-537, 2007.

REICHARDT, K.; TIMM, L. C. Solo, Planta e Atmosfera: Conceitos, processos e aplicações. Barueri-SP: Manole, 2004. $478 \mathrm{p}$.

SOUZA, G. B.; NOGUEIRA, A. R. D. A.; RASSINI, J. B. Determinação de matéria seca e umidade em solos e plantas com uso de forno de microondas doméstico. EMBRAPA: Embrapa Pecuária Sudeste, São Carlos-SP. 2002, 9p.

TOMÉ, J. B.; DECHEN, A. R. Microware Ovendrying of soil samples for chemical testing in Brazil. Communications in soil science and plants analysis. New York, v. 26, n. 3-4, p. 515-529, 1995. 